\title{
SECOND ORDER ACCURATE UPWIND SOLUTIONS OF THE 2D STEADY EULER EQUATIONS BY THE USE OF A DEFECT CORRECTION METHOD
}

\author{
S.P.Spekreijse \\ CWI, Centre for Mathematics and Computer Science \\ P.O.Box 4079, 1009 AB Amsterdam, The Netherlands
}

\begin{abstract}
In this paper a description is given of first and second order finite volume upwind schemes for the $2 \mathrm{D}$ steady Euler equations in generalized coordinates. These discretizations are obtained by projectionevolution stages, as suggested by Van Leer. The first order schemes can be solved efficiently by multigrid methods. Second order approximations are obtained by a defect correction method. In order to maintain monotone solutions, a limiter is introduced for the defect correction method.
\end{abstract}

\section{INTRODUCTION}

The steady state equations of inviscid flow, the steady Euler equations, are a nonlinear nonelliptic system of equations admitting solutions with discontinuities (shocks, contact discontinuities).

An important class of difference schemes for the (steady) Euler equations are the first order upwind schemes. These schemes are found by subdividing the domain of interest in disjunct control volumes (finite volume technique) and by assuming that the states in the volumes (or cells) are uniform (piecewise constant approximation). Then at each cell boundary two uniform states meet in a discontinuity. A unique flux, in the literature called the numerical flux [6], can be assigned after resolving the discontinuity by a set of elementary waves moving normal to the cell boundary or, in other words, after solving the one-dimensional Riemann-problem. This may be done exactly (Godunov [5]) or approximately (Osher [11], Roe [12], Van Leer [15], Steger \& Warming [13]). The only difference between these schemes is the way of approximating the Riemann-problem. Therefore each first order upwind scheme is characterized by its numerical flux function.

Succesful application of the multigrid method for the solution of the nonlinear system obtained by first order upwind schemes has been reported by Mulder \& Van Leer [10],[18] and Hemker \& Spekreijse [8],[9]. They use respectively Van Leer's and Osher's approximate Riemann-solver.

The purpose of this paper is to improve solutions of first order upwind schemes. This is highly 
desirable because solutions of these schemes have some important shortcomings. Because of the hidden viscosity in these schemes, oblique (with respect to the mesh) shocks and contact discontinuities are smeared out disastrously. Furthermore, in the smooth part of the flow field the solution is only first order accurate, which is too low for practical purposes. Therefore we wish to improve the order of accuracy and to steepen oblique discontinuities without introducing over- or undershoot.

In the literature many second order upwind schemes with flux limiters are available [14],[17],[3],[1]. Without a flux limiter solutions of second or higher order schemes suffer from oscillations in the neighbourhood of discontinuities. Therefore flux limiters have been constructed and implemented in these schemes to prevent these oscillations. Such schemes, with their time dependent term, belong to the class of total variation diminishing (TVD) schemes [14]. The steady state solution of a second order TVD scheme possesses the improvements we wish to obtain.

The construction of time dependent first or second order (TVD) upwind schemes can be considered most conveniently (as suggested by Van Leer [16],[1]) in two stages: a projection stage and an evolution stage (MUSCL-approach). In the projection stage the states in the cells are interpolated to yield approximations of the states at the cell boundaries. If this approximation is made by extrapolation from both sides of a cell boundary then two uniform states meet in a discontinuity. In the evolution stage, at each cell boundary, an approximate Riemann solver (or equivalently a numerical flux function) is used to calculate the flux from these two uniform states. When a second order upwind scheme is constructed by this method, a flux limiter, developed to make the scheme TVD, only needs to be applied in the projection stage.

Because the solution of first order upwind schemes can be obtained efficiently (by the multigrid method), it seems that an iterative defect correction (DeC) method provides a simple way to obtain the steady state solution of a second order TVD scheme. Unfortunately however, such an iterative $\mathrm{DeC}$ process will converge very slowly or might not even converge at all. On the other hand, it is well known [2] that just one or more $\mathrm{DeC}$ iterations are enough to obtain a second order accurate approximation. Therefore we shall apply the $\mathrm{DeC}$ iteration steps only a few times. This makes the method cheap to apply but it also implies that the steady state solution of the second order TVD scheme will not be achieved. The flux limiter, developed to make the second order upwind scheme TVD, only ensures that the steady state solution will be monotone and not that a second order approximation obtained after a few $\mathrm{DeC}$ iteration steps is monotone as well. It is practical experience that wiggles may occur after a few defect corrections despite the use of a flux limiter. Therefore in the context of the $\mathrm{DeC}$ method, it is not appropriate to use a flux limiter.

On the other hand, after each $\mathrm{DeC}$ iteration step the new solution can be considered as the sum of the solution of the first order upwind scheme plus a correction. In order to prevent that the addition of the correction to the first order solution creates new local extrema (wiggles), this correction has to be modified (limited!). The modification of the correction is one of the main topics of this paper. Because the first order upwind scheme is a monotone scheme, this strategy ensures that after each $\mathrm{DeC}$ iteration step the solution will stay monotone, or in other words no over- or undershoot will occur. Shortly, we suggest to apply a limiter in the $\mathrm{DeC}$ method and not in the second order upwind scheme.

In section 2 we describe the first and second order upwind discretization of the $2 \mathrm{D}$ steady Euler equations in general geometries. The subdivision of the discretization by the projection and evolution stages is applied. A proof is given of the accuracy of these discretizations.

In section 3 the DeC method is described. A description of a simple limiter used in the DeC method is given.

In section 4 some numerical results concerning the resolution of a contact discontinuity and an oblique shock are presented. 


\section{SECOND ORDER FINITE VOLUME UPWIND DISCRETIZATION OF THE 2D STEADY EULER EQUATIONS}

The 2D Euler equations can be written in conservative-vector form as

$$
\frac{\partial}{\partial t} q+\frac{\partial}{\partial x} f(q)+\frac{\partial}{\partial y} g(q)=0
$$

on an open (irregular) domain $\Omega^{*} \subset \mathbb{R}^{2}$, where

$$
q=\left(\begin{array}{c}
\rho \\
\rho u \\
\rho v \\
e
\end{array}\right), \quad f(q)=\left(\begin{array}{c}
\rho u \\
\rho u^{2}+p \\
\rho u v \\
u(e+p)
\end{array}\right), \quad g(q)=\left(\begin{array}{c}
\rho v \\
\rho u v \\
\rho v^{2}+p \\
v(e+p)
\end{array}\right) .
$$

Here, respectively $\rho, u, v, p$ and e are density, velocity components in the $\mathrm{x}$ - and $\mathrm{y}$-directions, pressure and total energy per unit volume. Furthermore, e may be expressed as

$$
e=\rho\left(\epsilon+1 / 2\left(u^{2}+v^{2}\right)\right),
$$

where the specific internal energy $\epsilon$, is related to the pressure and density by the perfect gas law

$$
p=(\gamma-1) \rho \epsilon
$$

with $\gamma$ denoting the ratio of specific heats.

The physical domain $\Omega^{*}$ is subdivided into disjunct quadrilateral cells $\Omega_{i, j}^{*},(i, j) \in\{1 . . M, 1 . . N\}$ in a regular fashion such that

i) $\Omega^{*}=\bigcup_{i, j} \Omega_{i, j}^{*}$,

ii) $\Omega_{i, j}^{*}, \Omega_{i \pm 1, j}^{*}, \Omega_{i, j \pm 1}^{*}$ are neighbouring cells,

iii) $\left(x_{i+1 / 2, j+1 / 2}, y_{i+1 / 2, j+1 / 2}\right)=\bar{\Omega}_{i, j}^{*} \cap \bar{\Omega}_{i+1, j}^{*} \cap \bar{\Omega}_{i, j+1}^{*} \cap \bar{\Omega}_{i+1, j+1}^{*}$ is a common vertex

of the cells $\Omega_{i, j}^{*}, \Omega_{i+1, j}^{*}, \Omega_{i, j+1}^{*}$ and $\Omega_{i+1, j+1}^{*}$.

It is clear that the vertices $\left\{\left(x_{i+1 / 2, j+1 / 2}, y_{i+1 / 2, j+1 / 2}\right)\right\}$ define the subdivision of $\Omega^{*}$ completely.

Let $(\xi, \eta)$ and $(x, y)$ denote the cartesian coordinates in respectively the computational and physical space. In the computational space, we consider a rectangular domain $\Omega$ subdivided into equidistant square control volumes (or cells) $\Omega_{i, j},(i, j) \in\{1 . . M, 1 . . N\}$ in such a way that $(h \cdot i, h \cdot j)$ is the midpoint of $\Omega_{i, j} ; h$ denotes the length of the edges. Assume the existence of a sufficiently smooth 1-1 relation between $(\xi, \eta)$ and $(x, y)$ :

$$
\left\{\begin{array} { l } 
{ \xi = \xi ( x , y ) } \\
{ \eta = \eta ( x , y ) }
\end{array} \Leftrightarrow \left\{\begin{array}{l}
x=x(\xi, \eta) \\
y=y(\xi, \eta)
\end{array},\right.\right.
$$

such that each cell $\Omega_{i, j}$ corresponds with $\Omega_{i, j}^{*}$ by this mapping i.e. for all $(i, j) \in\{0 . . M, 0 . . N\}$

$$
\left(x_{i+1 / 2, j+1 / 2}, y_{i+1 / 2, j+1 / 2}\right)=\left(x\left(\xi_{i+1 / 2}, \eta_{i+1 / 2}\right), y\left(\xi_{i+1 / 2}, \eta_{i+1 / 2}\right)\right),
$$

where $\xi_{i+1 / 2}=(i+1 / 2) \cdot h$ and $\eta_{j+1 / 2}=(j+1 / 2) \cdot h$. It can be easily seen that in the computational space $(\xi, \eta)$ the Euler equations become

$$
\frac{\partial}{\partial t}(J q)+\frac{\partial}{\partial \xi}\left(y_{\eta} f(q)-x_{\eta} g(q)\right)+\frac{\partial}{\partial \eta}\left(x_{\xi} g(q)-y_{\xi} f(q)\right)=0,
$$

with $J=x_{\xi} y_{\eta}-y_{\xi} x_{\eta}$.

The discretization of (2.1) on $\Omega^{*}$ is equivalent with the discretization of (2.7) on $\Omega$. Because $\Omega$ is a rectangle subdivided into equidistant cells of lenght $h$, it is easier to obtain first and second order 
upwind discretizations of (2.7) on $\Omega$ than of (2.1) on $\Omega^{*}$. In symbolic form we write (2.7) as

$$
(J q)_{t}+N(q)=0,
$$

and the steady Euler equations as

$$
N(q)=0,
$$

Here $N: X \rightarrow Y$ is a nonlinear operator, $X \subset\left[L^{2}(\Omega)\right]^{4}$ is the space of possible fluid states and $Y=\left[L^{2}(\Omega)\right]^{4}$ is the Banach space of rates of change (of states).

Define the finite dimensional vector spaces $X_{h}$ and $Y_{h}$ by

$$
X_{h}=Y_{h}=\left\{q_{i, j} \in \mathbb{R}^{4} \mid i=1 . . M, j=1 . . N\right\} .
$$

The relation between the spaces $X$ and $X_{h}, Y$ and $Y_{h}$ is obtained by introducing $R_{h}: X \rightarrow X_{h}$ and $\bar{R}_{h}: Y \rightarrow Y_{h}$

$$
\left(R_{h} q\right)_{i, j}=\left(\bar{R}_{h} q\right)_{i, j}=\frac{1}{h^{2}} \iint_{\Omega_{i, j}} q(\xi, \eta) d \xi d \eta,
$$

for any $q \in\left[L^{2}(\Omega)\right]^{4}$. Thus $\left(R_{h} q\right)_{i, j}$ is the mean value of $q$ in $\Omega_{i, j}$. A p-order accurate discretization of (2.9) is an associated problem

$$
N_{h}^{p}(q)=0,
$$

where $N_{h}^{p}: X_{h} \rightarrow Y_{h}$ has the property that for all sufficiently smooth $q \in X$

$$
\left(N_{h}^{p} R_{h} q\right)_{i, j}-\left(\bar{R}_{h} N q\right)_{i, j}=O\left(h^{p}\right) .
$$

In this paper we will consider first and second order upwind schemes, so $\mathrm{p}=1$ or $\mathrm{p}=2$.

Notice that

$$
\begin{aligned}
& \left(\bar{R}_{h} N q\right)_{i, j}=\frac{1}{h^{2}} \cdot\left\{\int_{\Gamma_{i+h, j}}\left(y_{\eta} f-x_{\eta} g\right)(q((i+1 / 2) \cdot h, \eta)) d \eta-\right. \\
& \int_{\Gamma_{i-h, j}}\left(y_{\eta} f-x_{\eta} g\right)(q((i-1 / 2) \cdot h, \eta)) d \eta+\int_{\Gamma_{i, j+\eta}}\left(x_{\xi} g-y_{\xi} f\right)(q(\xi,(j+1 / 2) \cdot h)) d \xi- \\
& \left.\int_{\Gamma_{i, j-h}}\left(x_{\xi} g-y_{\xi} f\right)(q(\xi,(j-1 / 2) \cdot h)) d \xi\right\},
\end{aligned}
$$

where $\Gamma_{i+1 / 2, j}, \Gamma_{i-1 / 2, j}, \Gamma_{i, j+1 / 2}, \Gamma_{i, j-1 / 2}$ denote the boundaries of cell $\Omega_{i, j}$, defined by $\Gamma_{i+1 / 2, j}=\bar{\Omega}_{i, j} \cap \bar{\Omega}_{i+1, j}, \Gamma_{i, j+1 / 2}=\bar{\Omega}_{i, j} \cap \bar{\Omega}_{i, j+1}$ etc.

Now we will construct an operator $\tilde{N}_{h}: X \rightarrow Y_{h}$, easier to approximate than $\bar{R}_{h} N$, but such that for all sufficiently smooth $q \in X$

$$
\left(\tilde{N}_{h} q\right)_{i, j}-\left(\bar{R}_{h} N q\right)_{i, j}=O\left(h^{2}\right) .
$$

If we are able to construct $N_{h}^{p}: X_{h} \rightarrow Y_{h}, p=1,2$ such that

$$
\left(N_{h}^{p} R_{h} q\right)_{i, j}-\left(\tilde{N}_{h} q\right)_{i, j}=O\left(h^{P}\right)
$$

then also

$$
\left(N_{h}^{p} R_{h} q\right)_{i, j}-\left(\bar{R}_{h} N q\right)_{i, j}=O\left(h^{p}\right) .
$$

This means that $\tilde{N}_{h}$ fter the construction of a $\tilde{N}_{h}$ satisfying (2.15) we may restrict ourselves to the approximation of $\tilde{N}_{h}$ instead of $\bar{R}_{h} N$. An obvious choice for the operator $\bar{N}_{h}$ is

$$
\left(\tilde{N}_{h} q\right)_{i, j}=\frac{1}{h} \cdot\left\{\left(y_{\eta_{i+h, j}} f-x_{\eta_{i+h_{j}}} g\right)\left(\bar{q}_{i+1 / 2, j}\right)-\left(y_{\eta_{i}-h_{, j}} f-x_{\eta_{i}-h_{, j}} g\right)\left(\bar{q}_{i-1 / 2, j}\right)\right.
$$




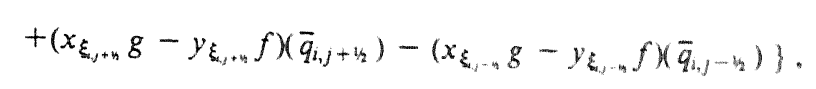

where

and

$$
\begin{aligned}
& y_{\eta_{i}+h_{, j}}=\frac{1}{h} \cdot\left(y_{i+1 / 2, j+1 / 2}-y_{i+1 / 2, j-1 / 2}\right), x_{\eta_{1}+n_{j, j}}=\frac{1}{h} \cdot\left(x_{i+1 / 2, j+1 / 2}-x_{i+1, j, j-y_{2}}\right), \\
& x_{\xi_{i, j}+n}=\frac{1}{h} \cdot\left(x_{i+1 / 2, j+1 / 2}-x_{i-1 / 2, j+1 / 2}\right), y_{\xi_{i,+4}}=\frac{1}{h} \cdot\left(y_{i+1 / 2, j+1 / 2}-y_{i-1 / 2, j+1 / 2}\right) \text {, }
\end{aligned}
$$

$$
\bar{q}_{i+1 / 2, j}=\frac{1}{h} \cdot \int_{\Gamma_{i+h, j}} q((i+1 / 2) \cdot h, \eta) d \eta .
$$

Thus $\bar{q}_{i+1 / 2, j}$ is the mean value of $q(\xi, \eta)$ at the cell boundary $\Gamma_{i+1,2, j}$. In a similar way $\bar{q}_{i-1 / 2, j}, \bar{q}_{i, j+1 / 2}$ and $\bar{q}_{i, j-1 / 2}$ are defined. By (2.18a) we see that the derivatives of the mapping, at the cell boundaries, are approximated by central differences.

By elementary interpolation theory it can be seen that $(2.15)$ holds (assuming a sufficiently smooth mapping).

We wish to deal with upwind schemes therefore we have to introduce an approximate Riemannsolver or equivalently a numerical flux function. This can be easily done by using the property of rotational invariance of the Euler equations i.e.

with

$$
\cos \phi \cdot f(q)+\sin \phi \cdot g(q)=T(\phi)^{-1} f(T(\phi) q),
$$

$$
T(\phi)=\left(\begin{array}{cccc}
1 & 0 & 0 & 0 \\
0 & \cos \phi & \sin \phi & 0 \\
0 & -\sin \phi & \cos \phi & 0 \\
0 & 0 & 0 & 1
\end{array}\right),
$$

and $q, f(q)$ and $g(q)$ as in (2.2), $\phi \in \mathbb{R}$.

Define $l_{i+1 / 2, j}, l_{i, j+1 / 2}$ by

$$
l_{i+1 / 2, j}=\left(y_{\eta_{i+n_{j}}}{ }^{2}+x_{\eta_{i+n_{j}}}\right)^{1 / 2}, l_{i, j+1 / 2}=\left(x_{\xi_{i,+n}}{ }^{2}+y_{\xi_{j,+m}}{ }^{2 / / 2},\right.
$$

and $\phi_{i+1 / 2, j}, \phi_{i, j+1 / 2}$ by

$$
\begin{array}{ll}
l_{i+1 /, j} \cdot \cos \phi_{i+1 / 2, j}=y_{\eta_{i+h_{j}}} & , l_{i+1 / 2, j} \cdot \sin \phi_{i+1 / 2, j}=-x_{\eta_{i+n_{j}}}, \\
l_{i, j+1 / 2} \cdot \cos \phi_{i, j+1 / 2}=-y_{\xi_{i,+n}} & , l_{i, j+y_{2}} \cdot \sin \phi_{i, j+1 / 2}=x_{\xi_{i, j+h}},
\end{array}
$$

then using (2.19)-(2.21), (2.18) yields

$$
\begin{aligned}
\left(\tilde{N}_{h} q\right)_{i, j}= & \frac{1}{h} \cdot\left\{l_{i+1 / 2, j} T_{i+1 / 2, j}^{-1} \cdot f\left(T_{i+1 / 2, j} \bar{q}_{i+1 / 2, j}\right)-l_{i-1 / 2, j} T_{i-1 / 2, j}^{-1} \cdot f\left(T_{i-1 / 2, j} \bar{q}_{i-1 / 2, j}\right)\right. \\
& \left.+l_{i, j+1 / 2} T_{i, j+1 / 2}^{-1} \cdot f\left(T_{i, j+1 / 2} \bar{q}_{i, j+1 / 2}\right)-l_{i, j-1 / 2} T_{i, j-1 / 2}^{-1} \cdot f\left(T_{i, j-1 / 2} \bar{q}_{i, j-l_{2}}\right)\right\},
\end{aligned}
$$

where $T_{i+1 / 2, j}=T\left(\phi_{i+1 / 2, j}\right)$ etc. This formula strongly suggest how to define the first and second order upwind schemes $N_{h}^{p}: X_{h} \rightarrow Y_{h}, p=1,2$ namely

$$
\begin{aligned}
& \left(N_{h}^{p} q\right)_{i, j}=\frac{1}{h} \cdot\left\{l_{i+1 / 2, j} T_{i+1 /, j}^{-1} f\left(T_{i+1 / 2, j} q_{i+1 / 2, j}^{-}, T_{i+1 / 2, j} q_{i+1 / 2, j}^{+}\right)-\right. \\
& l_{i-1 / 2, j} T_{i-1 / 2, j}^{-1} f\left(T_{i-1 / 2, j} q_{i-1 / 2, j}^{-}, T_{i-1 / 2, j} q_{i-1 / 2, j}^{+}\right)+l_{i, j+1 / 2} T_{i, j+1 / 2}^{-1} f\left(T_{i, j+1 / 2} q_{i, j+1 / 2}^{-}, T_{i, j+1 / 2} q_{i, j+1 / 2}^{+}\right)- \\
& \left.l_{i, j-1 / 2} T_{i, j-1 / 2}^{-1} f\left(T_{i, j-1 / 2} q_{i, j-1 / 2}^{-}, T_{i, j-1 / 2} q_{i, j}^{+}-1 / 2\right)\right\},
\end{aligned}
$$

where 


$$
\begin{aligned}
& q_{i+1 / 2, j}^{-}=q_{i, j}, q_{i+1 / 2, j}^{+}=q_{i+1, j}, \\
& q_{i, j+1 / 2}^{-}=q_{i, j}, q_{i, j+1 / 2}^{+}=q_{i, j+1},
\end{aligned}
$$

for the first order approximation $(p=1)$ or

$$
\begin{aligned}
& q_{i+1 / 2, j}^{-}=q_{i, j}+1 / 2 \cdot\left(q_{i, j}-q_{i-1, j}\right), q_{i+1 / 2, j}^{+}=q_{i+1, j}+1 / 2 \cdot\left(q_{i+1, j}-q_{i+2, j}\right), \\
& q_{i, j+1 / 2}^{-}=q_{i, j}+1 / 2 \cdot\left(q_{i, j}-q_{i, j-1}\right), q_{i, j+1 / 2}^{+}=q_{i, j+1}+1 / 2 \cdot\left(q_{i, j+1}-q_{i, j+2}\right),
\end{aligned}
$$

for the second order approximation $(p=2)$ and $q \in X_{h}$. Furthermore $f():, \mathbb{R}^{4} \times \mathbb{R}^{4} \rightarrow \mathbb{R}^{4}$ is one of the numerical flux functions found in the literature [5],[11],[12],[13],[15]. For consistency we only need that the numerical flux $f($,$) is consistent with the physical flux i.e.$

$$
f(q, q)=f(q)
$$

To proof that $\left(N_{h}^{p} R_{h} q\right)_{i, j}-\left(\tilde{N}_{h} q\right)_{i, j}=O\left(h^{p}\right), p=1,2$ it is easily seen that we only need to show that for all sufficiently smooth $q \in X$

$$
\begin{aligned}
& \frac{1}{h} \cdot\left[l_{i+1 / 2, j} T_{i+1 /, j}^{-1}\left\{f\left(T_{i+1 /, j} q_{i+1 / 2, j}^{-}, T_{i+1 /, j} q_{i+1 /, j}^{+}\right)-f\left(T_{i+1 /, j} \bar{q}_{i+1 /, j}\right)\right\}-\right. \\
& \left.\quad l_{i-1 / 2, j} T_{i-1 / 2, j}^{-1}\left\{f\left(T_{i-1 /, j} q_{i-1 / 2, j}^{-}, T_{i-1 /, j} q_{i-1 / 2, j}^{+}\right)-f\left(T_{i-1 /, j} \bar{q}_{i-1 / 2, j}\right)\right\}\right]=O\left(h^{P}\right),
\end{aligned}
$$

with the same conventions as in (2.18b) and (2.23); thus $q_{i, j}=\left(R_{h} q\right)_{i, j}$ in $(2.23 \mathrm{a}, \mathrm{b})$. In order to prove (2.25) first define

$$
\bar{q}(\xi, \eta)=\frac{1}{h^{2}} \cdot \int_{\xi-\frac{h}{2}}^{\xi+\frac{h}{2}} \int_{\eta-\frac{h}{2}}^{\eta+\frac{h}{2}} q(\alpha, \beta) d \alpha d \beta,
$$

then

$$
\bar{q}(i h, j h)=\frac{1}{h^{2}} \cdot \iint_{\Omega_{i, j}} q(\alpha, \beta) d \alpha d \beta=\left(R_{h} q\right)_{i, j},
$$

and

$$
\left.\frac{\partial}{\partial \xi} \bar{q}(\xi, \eta)\right|_{(i h, j h)}=\frac{1}{h} \cdot\left(\bar{q}_{i+1 / 2, j}-\bar{q}_{i-1 / 2, j}\right) .
$$

From (2.27) and (2.23a,b) we see that

$$
\frac{1}{h} \cdot\left(q_{i+1 / 2, j}^{+}-q_{i-1 / 2, j}^{+}\right)=\left.\frac{\partial}{\partial \xi} \bar{q}(\xi, \eta)\right|_{(i h, j h)}+O\left(h^{p}\right) .
$$

Thus, using (2.28), (2.29) yields

$$
q_{i+1 / 2, j}^{ \pm}-\bar{q}_{i+1 / 2, j}=q_{i-1 / 2, j}^{ \pm}-\bar{q}_{i-1 / 2, j}+O\left(h^{p+1}\right) .
$$

Furthermore it is clear that

$$
q_{i+1 / 2, j}^{ \pm}-\bar{q}_{i+1 / 2, j}=O\left(h^{p}\right)
$$

Assuming that the numerical flux function is sufficiently smooth, it follows by a Taylor expansion that

$$
\begin{aligned}
& f\left(q_{i+1 / 2, j}, q_{i+1 / 2, j}^{+}\right)=f\left(\bar{q}_{i+1 / 2, j}, \bar{q}_{i+1 / 2, j}\right)+f_{1}^{\prime}\left(\bar{q}_{i+1 / 2, j}, \bar{q}_{i+1 / 2, j}\right)\left(q_{i+1 / 2, j}^{-}-\bar{q}_{i+1 / 2, j}\right)+ \\
& f_{2}^{\prime}\left(\bar{q}_{i+1 / 2, j}, \bar{q}_{i+1 / 2, j}\right)\left(q_{i+1 / 2, j}^{+}-\bar{q}_{i+1 / 2, j}\right)+O\left(\left|q_{i+1 / 2, j}^{-}-\bar{q}_{i+1 / 2, j}\right|^{2},\left|q_{i+1 / 2, j}^{+}-\bar{q}_{i+1 / 2, j}\right|^{2}\right) .
\end{aligned}
$$

Due to the consistency of the numerical flux function and using (2.31), (2.32) yields

$$
f\left(T_{i+1 / 2, j} q_{i+1 / 2, j}^{-}, T_{i+1 / 2, j} q_{i+1 / 2, j}^{+}\right)-f\left(T_{i+1 / 2, j} \bar{q}_{i+1 / 2, j}\right)=
$$




$$
\begin{aligned}
& f^{\prime}{ }_{1}\left(T_{i+1 / 2, j} \bar{q}_{i+1 / 2, j}, T_{i+1 / 2, j} \bar{q}_{i+1 / 2, j}\right) \cdot T_{i+1 / 2, j} \cdot\left(q_{i+1 / 2, j}-\bar{q}_{i+1 / 2, j}\right)+ \\
& f^{\prime}{ }_{2}\left(T_{i+1 / 2, j} \bar{q}_{i+1 / 2, j}, T_{i+1 / 2, j} \bar{q}_{i+1 / 2, j}\right) \cdot T_{i+1 / 2, j} \cdot\left(q_{i+1 / 2, j}^{+}-\bar{q}_{i+1 / 2, j}\right)+O\left(h^{2 p}\right) .
\end{aligned}
$$

If the mapping is sufficiently smooth then

$$
l_{i+1 / 2, j}-l_{i-1 / 2, j}=O(h), T_{i+1 / 2, j}-T_{i-1 / 2, j}=O(h), T_{i+1 / 2, j}^{-1}-T_{i-1 / 2, j}^{-1}=O(h) .
$$

From (2.33),(2.30),(2.31) and (2.34) it is easily derived that (2.25) holds. Hence, $N_{h}^{p}, \mathrm{p}=1,2$ are porder accurate approximations of $\mathrm{N}$.

\section{Remarks}

-The smoothness of the coordinate transformation (2.5) is necessary to obtain first and second order accurate space discretizations.

-The equation $\left(N_{h}^{P} q\right)_{i, j}=0$ is equivalent with $\left(h^{2} N_{h}^{p} q\right)_{i, j}=0$. After multiplication of (2.23) by $h^{2} \quad$ it is clear that $\left(h^{2} N_{h}^{p} q\right)_{i, j}$ is determined completely by the coordinates $\left\{\left(x_{i+1 / 2, j+1 / 2}, y_{i+1 / 2, j+1 / 2}\right)\right\}$ of the vertices of the mesh in the physical space (and, of course, by the numerical flux function $\mathrm{f}()$,$) ). This means that in the physical space on an irregular mesh, a first$ and second order finite volume scheme can be constructed without the actual need of a coordinate transformation. The finite volume space discretization on an irregular mesh behaves in a first or second order manner only if the irregular mesh is chosen in accordance with a smooth transformation.

-Consider the steady Euler equations in the computational space $N(q)=0$ and their discretization $\left(N_{h}^{p} q\right)_{i, j}=0, p=1,2$ (see respectively (2.9),(2.23)). Let $q^{p} \in X_{h}, p=1,2$ denote the solutions of the discrete equations and $q=\tilde{q}(\xi, \eta) \in X$ be the solution of the continuous equation. If the operators $N_{h}^{p}, p=1,2$ are stable then

$$
q_{i, j}^{p}=\bar{q}_{i, j}+O\left(h^{P}\right),
$$

where $\bar{q}_{i, j}=\left(R_{h} \tilde{q}\right)_{i, j}=\frac{1}{h^{2}} \cdot \iint_{\Omega_{i, j}} \tilde{q}(\xi, \eta) d \xi d \eta$. Define

$$
\bar{Q}_{i, j}=\frac{1}{V_{i, j}} \cdot \iint_{\Omega_{i, j}} \tilde{q}(\xi, \eta) \cdot J(\xi, \eta) d \xi d \eta,
$$

with

$$
V_{i, j}=\iint_{\Omega_{i, j}} J(\xi, \eta) d \xi d \eta
$$

Then $V_{i, j}$ is the area of $\Omega_{i, j}^{*}$ in the physical space and $\bar{Q}_{i, j}$ is the mean value of $\tilde{q}(x, y)$ in $\Omega_{i, j}^{*}$. Assuming sufficient smoothness of the Jacobian $J(\xi, \eta)$ it is easily seen that

$$
\frac{1}{h^{2}} \cdot \iint_{\Omega, J} J(\xi, \eta) \tilde{q}(\xi, \eta) d \xi d \eta=\frac{1}{h^{2}} \cdot \iint_{\Omega, j} J(\xi, \eta) d \xi d \eta \cdot \frac{1}{h^{2}} \cdot \iint_{\Omega_{1, j}} \tilde{q}(\xi, \eta) d \xi d \eta+O\left(h^{2}\right) .
$$

So, $\bar{Q}_{i, j}=\bar{q}_{i, j}+O\left(h^{2}\right)$ and (2.35) yields $q_{i, j}^{p}=\bar{Q}_{i, j}+O\left(h^{p}\right), p=1,2$. Therefore, $q_{i, j}^{p}$ may be considered as a p-order accurate approximation of $\bar{q}_{i, j}$ as well as $\bar{Q}_{i, j}$.

-In the literature [16],[4],[1] one encounters generalizations of (2.23b) namely

$$
\begin{aligned}
& q_{i+1 / 2, j}^{-}=q_{i, j}+\frac{1+\kappa}{4} \cdot\left(q_{i+1, j}-q_{i, j}\right)+\frac{1-\kappa}{4} \cdot\left(q_{i, j}-q_{i-1, j}\right), \\
& q_{i+1 / 2, j}^{+}=q_{i+1, j}+\frac{1+\kappa}{4} \cdot\left(q_{i, j}-q_{i+1, j}\right)+\frac{1-\kappa}{4} \cdot\left(q_{i+1, j}-q_{i+2, j}\right) .
\end{aligned}
$$

and similar $q_{i, j+1 / 2}^{+}, \kappa \in[-1,1]$.

It can be easily seen that (2.29) and (2.31) hold for each $\kappa$. Therefore (2.36) results in a second 
order accurate space discretization as well as (2.23b).

-Even when $x(\xi, \eta)=\xi, y(\xi, \eta)=\eta$ it can be seen that for all smooth $q \in X$

$$
\left(\tilde{N}_{h} q\right)_{i, j}-\left(R_{h} N q\right)_{i, j}=O\left(h^{2}\right) .
$$

So it is plausible that $R_{h} N$ cannot be approximated more accurately than second order if the flux computation is based on the calculation of constant states at the cell boundaries. This is due to the fact that at a cell boundary the mean flux differs from the flux calculated in the mean state. So this result is typical for 2 and 3 dimensional problems. A formal proof of this result can be given.

\section{THE DEFECT CORRECTION METHOD}

In section 2 the description of first and second order upwind space discretizations of the 2D steady Euler equations has been given.

In this section the steady Euler equations and their first and second order space discretizations are denoted by

$$
N q=r ; N_{h}^{1} q_{h}=r_{h} ; N_{h}^{2} q_{h}=r_{h},
$$

with $h$ the meshsize of the (finest) grid.

Let $q_{h}^{1^{*}}$ and $q_{h}^{2^{*}}$ be the solutions of respectively $N_{h}^{1} q_{h}=r_{h}$ and $N_{h}^{2} q_{h}=r_{h}$. Then $q_{h}^{1^{*}}$ can be calculated efficiently by the multigrid method. We wish to use the defect correction method and the second order space discretization operator $N_{h}^{2}$ to improve $q_{h}^{l^{*}}$.

In a first glance the iterative defect correction $(\mathrm{DeC})$ method seems applicable:

$$
\left\{\begin{array}{l}
q_{h}^{1}:=q_{h}^{1^{*}}, \\
N_{h}^{1} q_{h}^{n+1}=N_{h}^{1} q_{h}^{n}+\left(r_{h}-N_{h}^{2} q_{h}^{n}\right) \quad n=1,2 \ldots
\end{array}\right.
$$

Unfortunately, dealing with the steady Euler equations, this iteration process is impractical because of the following reasons:

- Suppose, for the moment, that $N, N_{h}^{1}$ and $N_{h}^{2}$ are linear scalar operators with $N_{h}^{1}$ and $N_{h}^{2}$ first and second order discretizations of $N$. Then the symbols $N(\omega), N_{h}^{1}(\omega)$ and $N_{h}^{2}(\omega)$ of respectively $N, N_{h}^{1}$ and $N_{h}^{2}$ are defined by

$$
N\left(e^{i \omega x}\right)=N(\omega) \cdot e^{i \omega x} ; N_{h}^{1,2}\left(e^{i \omega x}\right)=N_{h}^{1,2}(\omega) \cdot e^{i \omega x},
$$

and the accuracy of $N_{h}^{1}$ and $N_{h}^{2}$ can be expressed by

$$
N(\omega)-N_{h}^{l}(\omega)=O\left(h^{p_{1}}\right) ; N(\omega)-N_{h}^{2}(\omega)=O\left(h^{p_{2}}\right)
$$

( $\omega$ fixed).

If we define the error $v_{h}^{n}=q_{h}^{2 *}-q_{h}^{n}$ then

$$
N_{h}^{1} v_{h}^{n+1}=\left(N_{h}^{1}-N_{h}^{2}\right) v_{h}^{n} \text {. }
$$

Taking $v_{h}^{n}=e^{i \omega x}$ then

$$
v_{h}^{n+1}=\left\{1-\frac{N_{h}^{2}(\omega)}{N_{h}^{1}(\omega)}\right\} \cdot v_{h}^{n} .
$$

On a fixed grid with $\omega \rightarrow 0$, we see that the amplification factor goes to zero (due to the consistency of $N_{h}^{1}$ and $N_{h}^{2}$ ). This means that low frequency error components are damped effectively by the iterative $\mathrm{DeC}$ method. But for high frequency error components $\left(\frac{\pi}{2 h} \leqslant \omega \leqslant \frac{\pi}{h}\right)$ the difference 
between $N_{h}^{1}(\omega)$ and $N_{h}^{2}(\omega)$ can be quite large, causing slow or no convergence of the iteration process. Indeed, dealing with the Euler equations it is a practical experience that (3.2) converges very slowly or even not at all. Thus we may not expect to reach the fixed point $q_{h}^{2^{*}}$ of (3.2). Therefore it makes no sense to make $N_{h}^{2}$ TVD (by a fluxlimiter) because the TVD property only ensures that $q_{h}^{2 *}$ is wiggle free.

On the other hand, after one defect correction iteration $q_{h}^{2}$ obeys

$$
N_{h}^{d c} q_{h}^{2}:=N_{h}^{1}\left(2 N_{h}^{1}-N_{h}^{2}\right)^{-1} N_{h}^{1} q_{h}^{2}=r_{h} .
$$

Let $N_{h}^{d c}(\omega)$ denote the symbol of $N_{h}^{d c}$. Then

$$
N_{h}^{d c}(\omega)=\frac{\left\{N_{h}^{1}(\omega)\right\}^{2}}{2 N_{h}^{1}(\omega)-N_{h}^{2}(\omega)},
$$

and it is easily seen that

$$
\begin{aligned}
N(\omega)-N_{h}^{d c}(\omega) & =\frac{N(\omega) \cdot\left\{N(\omega)-N_{h}^{2}(\omega)\right\}-\left\{N(\omega)-N_{h}^{1}(\omega)\right\}^{2}}{N(\omega)-2 \cdot\left\{N(\omega)-N_{h}^{1}(\omega)\right\}+\left\{N(\omega)-N_{h}^{2}(\omega)\right\}} \\
& =O\left(h^{\min \left(2 p_{1}, p_{2}\right)}\right)
\end{aligned}
$$

Because $p_{1}=1$ and $p_{2}=2$, this implies that $N_{h}^{d c}$ is a second order space discretization of $N$. So $q_{h}^{2}$ is a second order accurate approximation. In the same way it can be seen that all $q_{h}^{2}, n \geqslant 2$ are second order accurate approximations. Therefore, one or more $\mathrm{DeC}$ iteration steps are sufficient to improve the order of accuracy. This is a well known result which also holds for nonlinear problems [2].

From these arguments it is clear that we will apply the DeC method just a few times (at most four iteration steps). Then we will obtain a second order accurate approximation which suffers from wiggles in the neighbourhood of discontinuities. Because (3.2) starts with the solution of a first order scheme, which is a monotone scheme, we can prevent these wiggles by looking more closely to the correction $\delta q_{h}^{n}=q_{h}^{n}-q_{h}^{1^{*}}$. After each iteration in (3.2) we will change $\delta q_{h}^{n}$, as less as possible, but such that adding the changed correction to $q_{h}^{\frac{1}{*}}$ no new local extrema are created. In this way no wiggles can be introduced. This changing of $\delta q_{h}^{n}$ is in fact a limiting process, therefore we may speak of a limited defect correction process. A simple limited defect correction process is:

step1: $\tilde{q}_{h}:=q_{h}^{*}$.

step2:

- Calculate $q_{h}$ from

$$
N_{h}^{1} q_{h}=N_{h}^{1} \tilde{q}_{h}+\left(r_{h}-N_{h}^{2} \tilde{q}_{h}\right) .
$$

- Calculate the correction

$$
\delta q_{h}:=q_{h}-q_{h}^{*},
$$

and set

$$
q_{h}:=q_{h}^{1^{*}}
$$

Then $q_{h}+\delta q_{h}$ is the solution of (3.3). Now change $q_{h}$ and $\delta q_{h}$ by scanning the grid in several directions (for instance from north-east to south-west and vice versa and from north-west to south-east and vice versa) and change the states $q_{h_{i, j}}$ and $\delta q_{h_{t,}}$ in the visiting control volume $\Omega_{i, j}$ by the following algorithm:

$$
q_{h_{i, j}}:=q_{h_{t,}}+\delta q_{h}^{\text {new }}{ }_{i, j},
$$




$$
\delta q_{h_{t,}}:=\delta q_{h_{i, j}}-\delta q_{h}^{\text {new }}{ }_{i, j},
$$

where

$$
\begin{aligned}
\delta q_{h}^{\text {new }}{ }_{i, j}= & H\left(\delta q_{h_{i, j}}\right) \cdot \min \left(\delta q_{h_{i, j}},\left|\delta q_{h}^{+}{ }_{i, j}\right|\right)+ \\
& +\left\{1-H\left(\delta q_{h_{i, j}}\right)\right\} \cdot \max \left(\delta q_{h_{i,}},-\left|\delta q_{h}^{-}{ }_{i, j}\right|\right)
\end{aligned}
$$

and

$$
\begin{aligned}
& \delta q_{h, j}^{+}:=\max \left(q_{h_{i-1, j}}, q_{h_{i+1, j}}, q_{h_{i, j-1}}, q_{h_{i j+1}}\right)-q_{h_{i, j}}, \\
& \delta q_{h_{i, j}}^{-}:=\min \left(q_{h_{i-1, j}}, q_{h_{i+1, j}}, q_{h_{i, j-1}}, q_{h_{i, j+1}}\right)-q_{h_{i, j}},
\end{aligned}
$$

and $H: \mathbb{R} \rightarrow \mathbb{R}$ denotes the Heaviside function

$$
H(x)=\left\{\begin{array}{ll}
1 & \text { if } x>0 \\
0 & \text { if } x<0
\end{array} .\right.
$$

- Finally set $\tilde{q}_{h}:=q_{h}$.

Formula (3.5)-(3.7) must be applied to each component of $q_{h_{t,}}$ and $\delta q_{h_{1,}}$ separately.

It can be easily seen that $\delta q_{h}^{\text {new }}{ }_{i, j}$ is a smooth function of $\delta q_{h_{t, j}}$ and $q_{h_{t, j}}$.

Furthermore it is clear that in this way no new local extrema will be introduced in a visiting control volume. Although it is possible that after changing the state in the visiting control volume $\Omega_{i, j}$, a local extrema is created in one of the four neighbouring cells $\Omega_{i \pm 1, j}, \Omega_{i, j \pm 1}$. This limiter neglects this possibility, which is expected to occur rarely.

Step 2 may be repeated a few times to steepen discontinuities effectively. In general, the limiter will not work in the smooth parts of the flow field, so in those parts the correction $\delta q_{h}$ will be added to $q_{h}$ completely. Only in the neighbourhood of discontinuities this limiter will do its job; preventing oscillations. Therefore this limiter also provides a way to detect discontinuities in the flow field.

\section{NUMERICAL RESULTS}

The numerical flux function used in these experiments is constructed with Osher's approximate Riemann-solver. Hence, both the first and second order upwind scheme are able to capture shocks and contact discontinuities. To see the improvement of the capturing property after a few DeC iteration steps, two model problems are considered. Problem 1 concerns an oblique shock reflected from a flat plate and problem 2 concerns an oblique contact discontinuity generated by the boundary conditions. The precise description of these two problems is:

Problem1: The oblique shock.

The domain $\Omega^{*}$ is $(0,4) \times(0,1)$. The exact solution has 3 subregions with uniform states as given in figure 4.1 . 


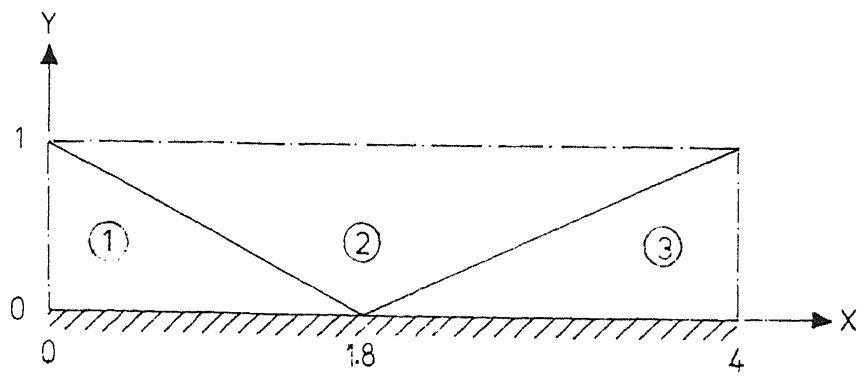

Figure 4.1.

The states are respectively:

state $1: u=2.9, v=0.0, c=1.0, p=1.0$,

state $2: u=2.6, v=-0.5, c=1.1, p=2.1$,

state $3: u=2.4, v=0.0, c=1.2, p=4.0$.

Problem2: The contact discontinuity.

Here $\Omega^{*}=(0,2) \times(0,2)$. The exact solution of the problem has a discontinuity at

$x+y=2$. In both parts of the domain the solution has a uniform state:

for $x+y<2$ we take $p=1.0, u=0.3, v=-0.3, c=0.6$,

for $x+y>2$ we take $p=1.0, u=0.6, v=-0.6, c=1.0$.

For the treatment of the boundary conditions see [7].

The figures 1,2,3 and 4 concern the resolution of the oblique shock and the figures 5 and 6 of the contact discontinuity.

Figure $1 \mathrm{a}, 1 \mathrm{~b}$ and $1 \mathrm{c}$ show the pressure contours on a $8 \times 24$ mesh, respectively obtained by the first order Osher scheme and after 1 and $4 \mathrm{DeC}$ iteration steps. Figure $2 \mathrm{a}, 2 \mathrm{~b}$ and $2 \mathrm{c}$ show the same results but on a $16 \times 48$ mesh. In all cases the limiter, described in section 3, has been used. In figure $3 a$ and $3 \mathrm{~b}$ pressure distributions along the flat plate are shown (using the $16 \times 48 \mathrm{mesh}$ ). In these figures results are shown, again obtained by the first order scheme and after 1 and $4 \mathrm{DeC}$ iterations. Figure $3 \mathrm{a}$ has been obtained with, figure $3 \mathrm{~b}$ without the limiter. Figure $4 \mathrm{a}$ and $4 \mathrm{~b}$ show similar results at $y=0.5$. After $4 \mathrm{DeC}$ iteration steps the quality of the shock capturing seems comparable with the results obtained by a second order TVD scheme [3].

Figure $5 \mathrm{a}, 5 \mathrm{~b}, 5 \mathrm{c}$ and $6 \mathrm{a}, 6 \mathrm{~b}, 6 \mathrm{c}$ show density contours on respectively a $16 \times 16$ and a $32 \times 32$ mesh. Again results of the first order scheme and after 1 and $4 \mathrm{DeC}$ iteration steps are shown. For comparison see [7].

For both problems, it is clear that after a few DeC iteration steps the capturing of the discontinuities has been improved considerably.

\section{CONCLUSION}

This paper is concerned with the discretization of the steady Euler equations by the finite volume technique. On an irregular mesh it is shown in detail how to apply Van Leer's projection-evolution stages in the discretization. Herein, the rotational invariance of the Euler equations is effectively used. For a general numerical flux function, consistent with the physical flux, a proof is given of the order of accuracy for a first and second order upwind scheme. Hence, the results hold for all well known approximate Riemann-solvers.

Second order accurate approximations are obtained by a defect correction $(\mathrm{DeC})$ method. A limiter, used in the DeC method, is constructed to maintain monotone solutions. For two typical model problems (an oblique shock and a contact discontinuity), only a few (3 or 4) DeC iteration steps are 
sufficient to steepen discontinuities effectively. This makes the method cheap to apply.Furthermore, the quality of the results seems comparable with results obtained by TVD schemes.

Acknowledgement. The author would like to thank P.W.Hemker, B.Koren and P.M.de Zeeuw for their cooperation and valuable suggestions.

\section{REFERENCES}

[1] Anderson, W.T., Thomas, J.L., and Van Leer, B., "A comparison of finite volume flux vector splittings for the Euler equations" AIAA Paper No. 850122.

[2] Böhmer, K., Hemker, P. \& Stetter, H., "The Defect Correction Approach." Computing Suppl. 5 (1984) $1-32$.

[3] Chakravarthy, S.R. and Osher, S., "High resolution applications of the Osher upwind scheme for the Euler equations." AIAA Paper 83-1943,Proc.AIAA Sixth Computational Fluid Dynamics Conf.(Danvers,Mass.July 1983), 1983,pp363-372.

[4] Chakravarthy, S.R. and Osher, S., "A new class of high accuracy TVD schemes for hyperbolic conservation laws." AIAA Paper 85-0363,AIAA 23rd Aerospace Science Meeting. (Jan.1417,1985/Reno,Nevada).

[5] Godunov, S.K., "A finite difference method for the numerical computation of discontinuous solutions of the equations of fluid dynamics." Mat.Sb.(N.S.)47(1959),271-;also Cornell Aeronautical Laboratory transl..

[6] Harten, A., Lax, P.D. \& Van Leer, B., "On upstream differencing and Godunov-type schemes for hyperbolic conservation laws." SIAM Review 25 (1983) 35-61.

[7] Hemker, P.W., "Defect correction and higher order schemes for the multi grid solution of the steady Euler equations." In this volume.

[8] Hemker, P.W. \& Spekreijse, S.P., "Multigrid solution of the Steady Euler Equations." In: Advances in Multi-Grid Methods (D.Braess, W.Hackbusch and U.Trottenberg eds) Proceedings Oberwolfach Meeting, Dec. 1984, Notes on Numerical Fluid Dynamics, Vol.11, Vieweg, Braunschweig, 1985.

[9] Hemker, P.W. \& Spekreijse, S.P., "Multiple Grid and Osher's Scheme for the Efficient Solution of the the Steady Euler Equations." Report NM-8507, CWI, Amsterdam, 1985.

[10] Mulder, W.A. "Multigrid Relaxation for the Euler equations." To appear in: J. Comp. Phys. 1985.

[11] Osher, S \& Solomon, F., "Upwind difference schemes for hyperbolic systems of conservation laws." Math. Comp. 38 (1982) 339-374.

[12] Roe, P.L., "Approximate Riemann solvers, parameter vectors and difference schemes." J. Comp. Phys. 43 (1981) 357-372.

[13] Steger, J.L. \& Warming, R.F., "Flux vector splitting of the inviscid gasdynamics equations with applications to finite difference methods." J. Comp. Phys. 40 (1981) 263-293.

[14] Sweby, P.K. "High resolution schemes using flux limiters for hyperbolic conservation laws", SIAM J.Numer.Anal. 21 (1984) 995-1011.

[15] Van Leer, B., "Flux-vector splitting for the Euler equations." In: Procs. 8th Intern. Conf. on numerical methods in fluid dynamics, Aachen, June, 1982. Lecture Notes in Physics 170, Springer Verlag.

[16] Van Leer, B., "Upwind difference methods for aerodynamic problems governed by the Euler equations." Report 84-23, Dept. Math. \& Inf., Delft Univ. Techn., 1984.

[17] Van Leer, B., "Towards the ultimate conservative difference scheme.2. Monotonicity and conservation combined in a second order scheme." J.Comp.Phys.14,361-370(1974).

[18] Van Leer, B. \& Mulder, W.A., "Relaxation methods for hyperbolic equations." Report 84-20, Dept. Math. \& Inf., Delft Univ. Techn., 1984. 


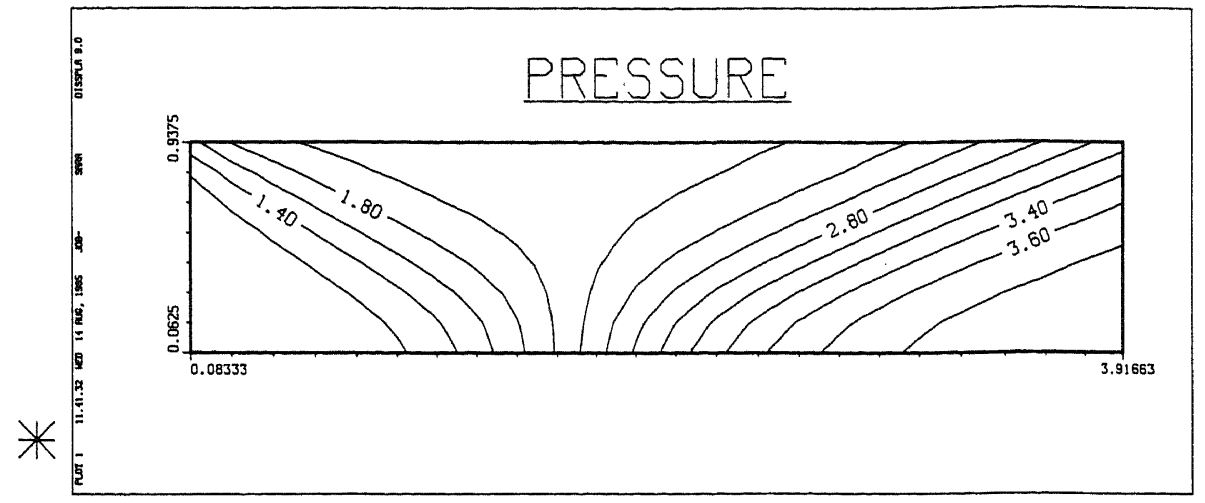

Figure 1a.

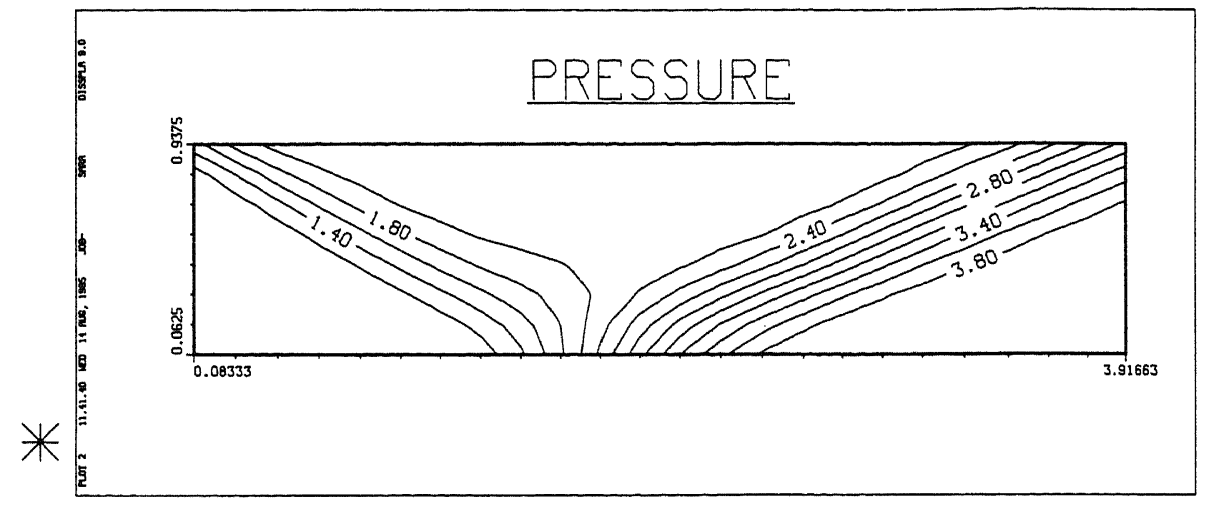

Figure lb.

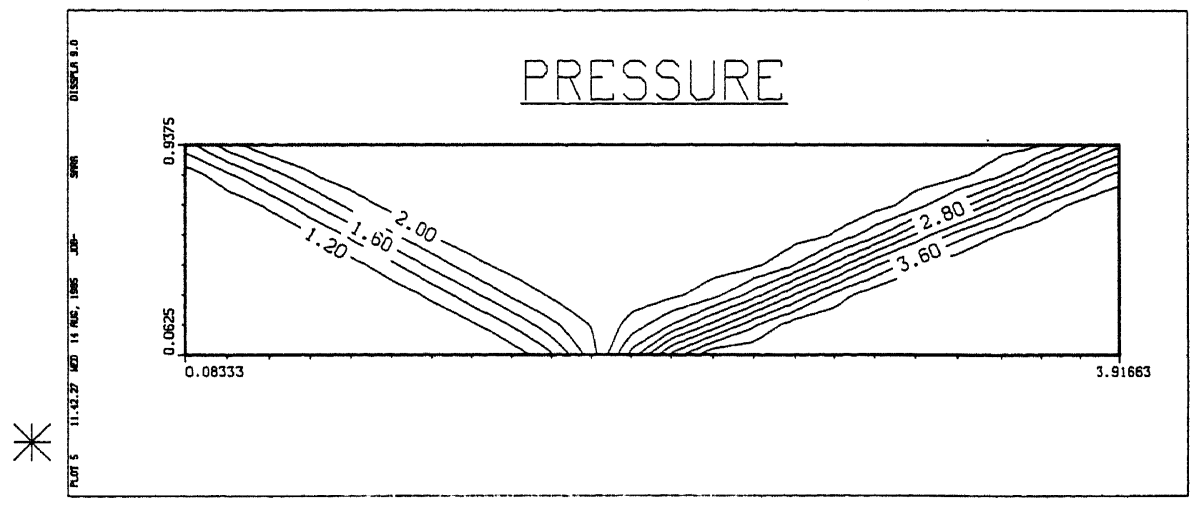

Figure 1c.

Pressure contours of an oblique shock on a $8 \times 24$ mesh, obtained by the first order upwind scheme and after 1 and $4 \mathrm{DeC}$ iteration steps. 


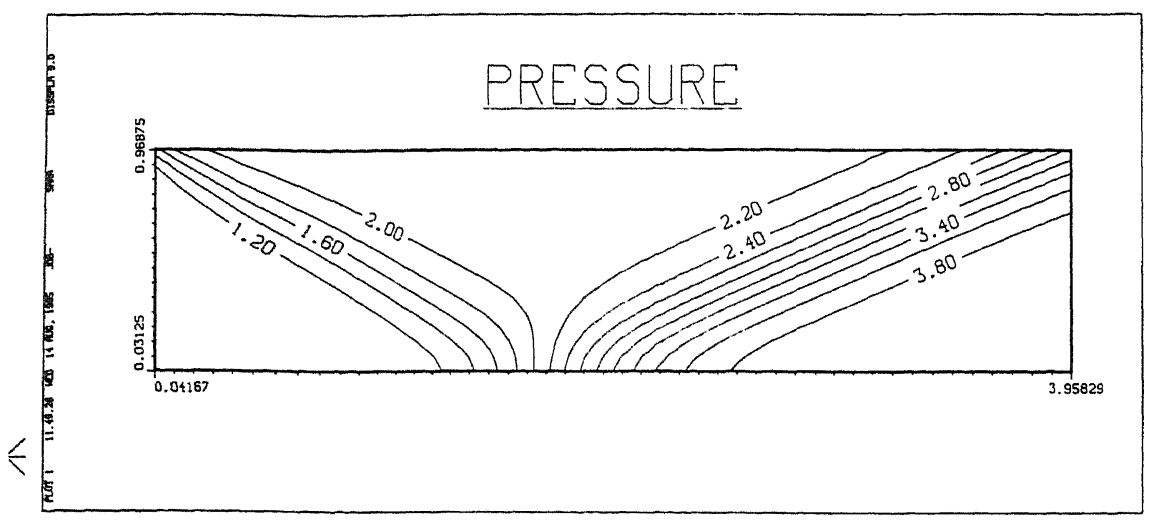

Figure $2 \mathrm{a}$.

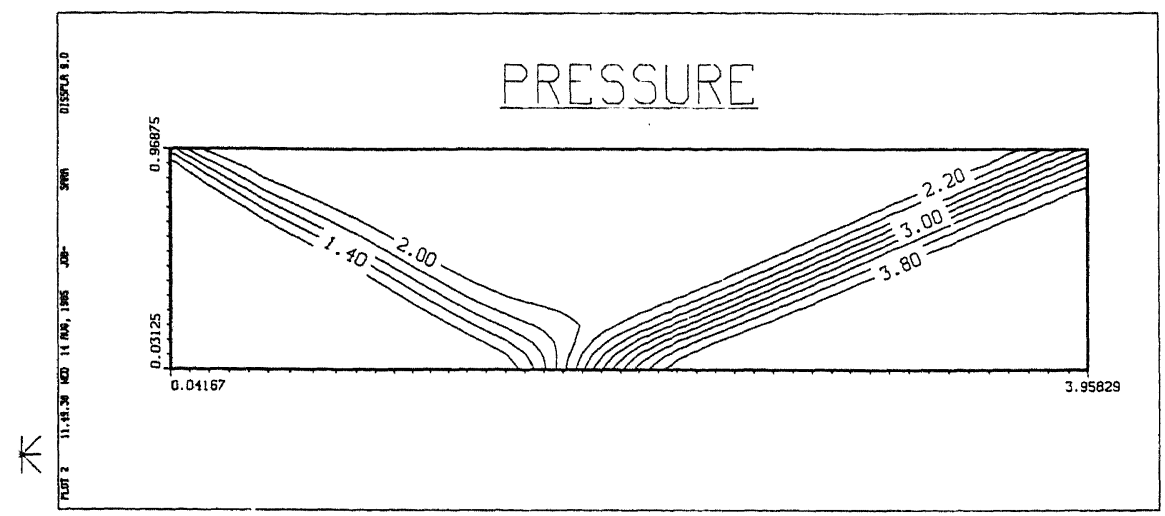

Figure 2b.



Figure 2c.

Pressure contours on a $16 \times 48$ mesh. 


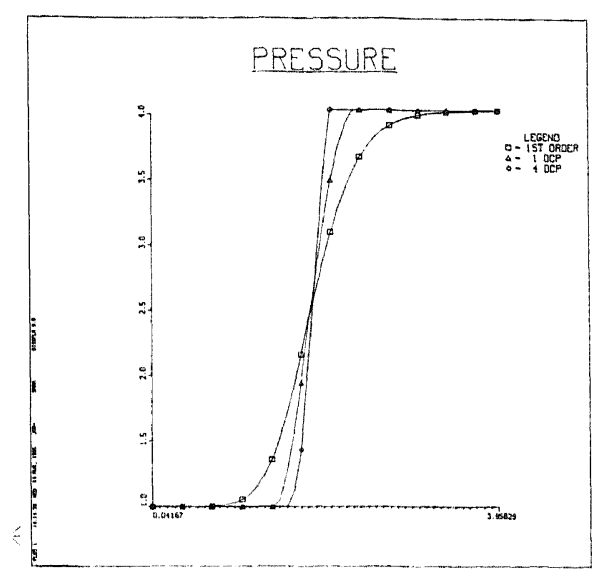

Figure 3a.

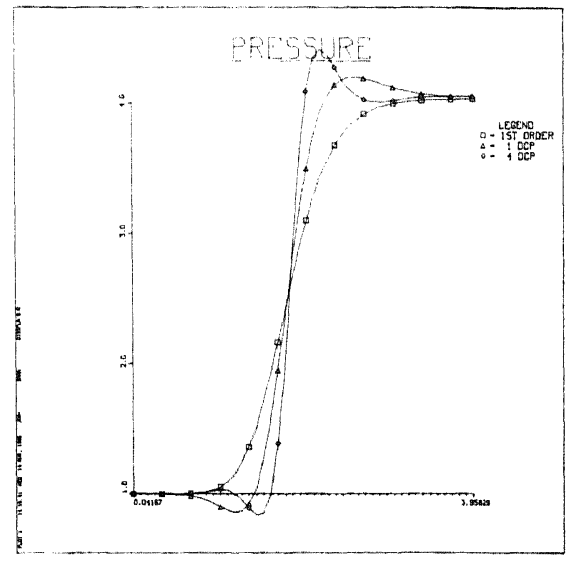

Figure 3b.

Pressure profiles computed at the surface of the flat plate, using the first order scheme and after 1 and $4 \mathrm{DeC}$ iteration steps. Figure $3 \mathrm{a}$ has been obtained with a limiter, figure $3 \mathrm{~b}$ without.

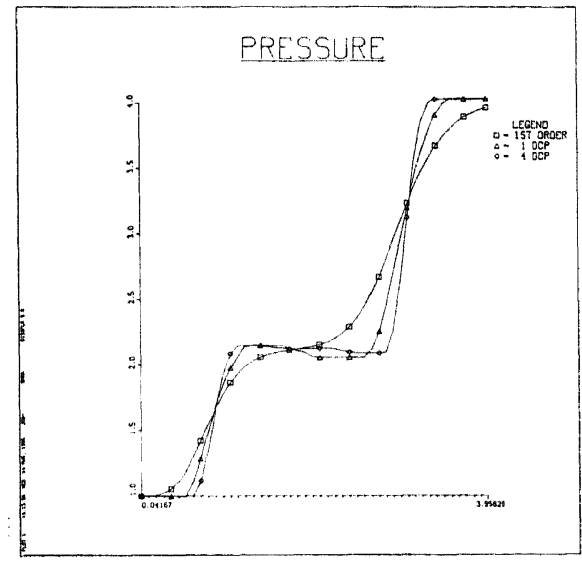

Figure 4a.

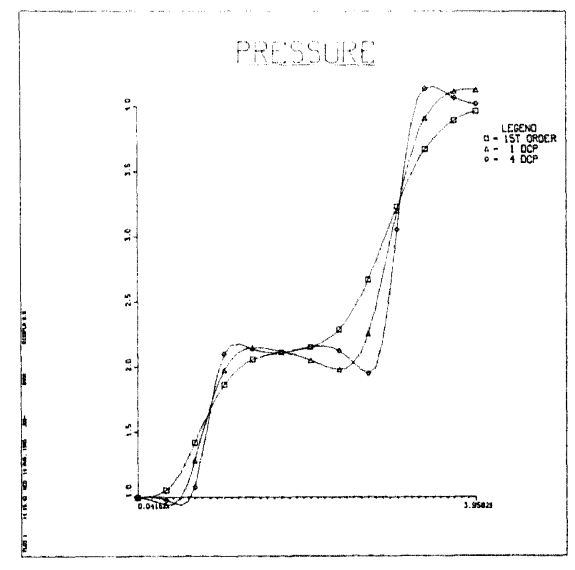

Figure $4 b$.

Pressure profiles at $y=0.5$. 


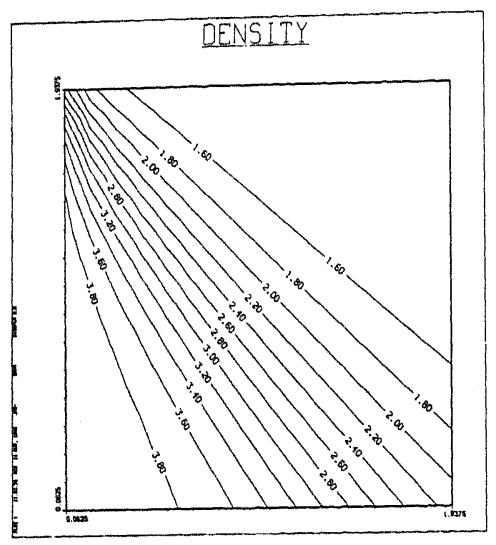

Figure 5a.

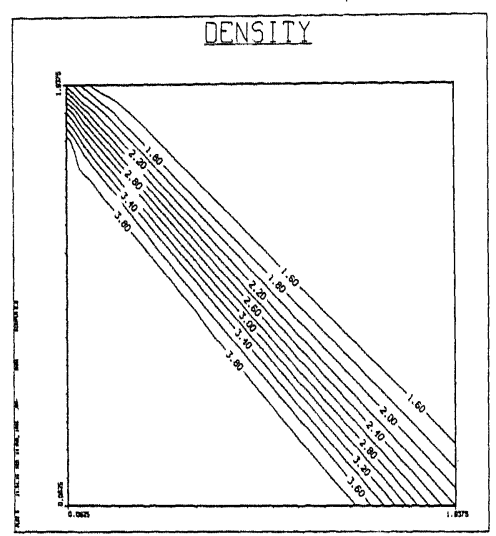

Figure 5c

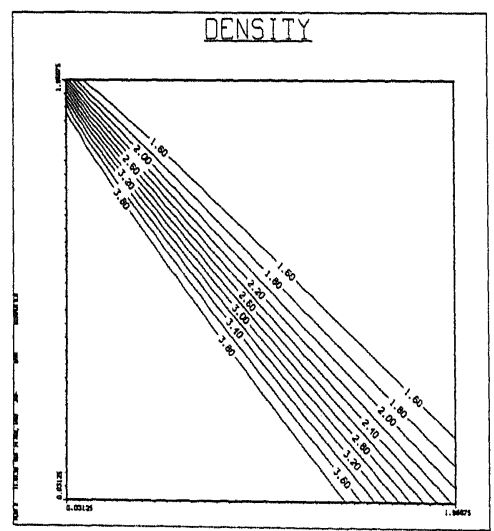

Figure $6 \mathrm{~b}$.

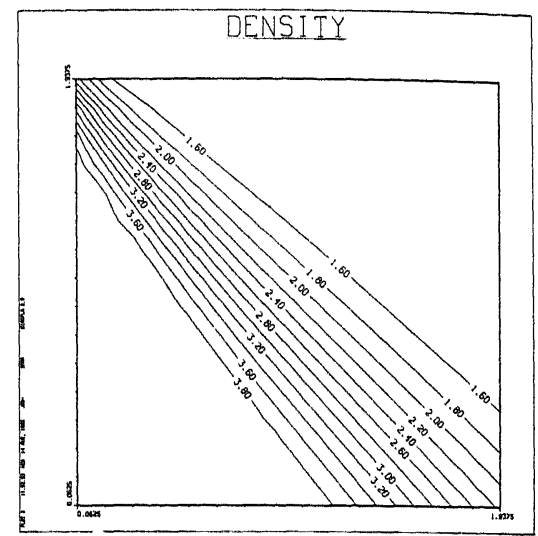

Figure 5b.

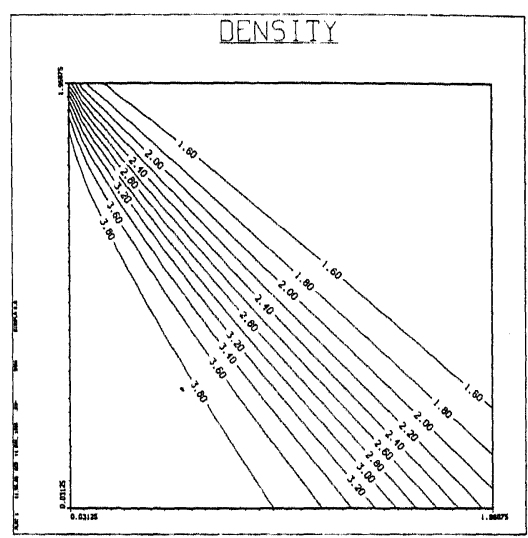

Figure 6a.

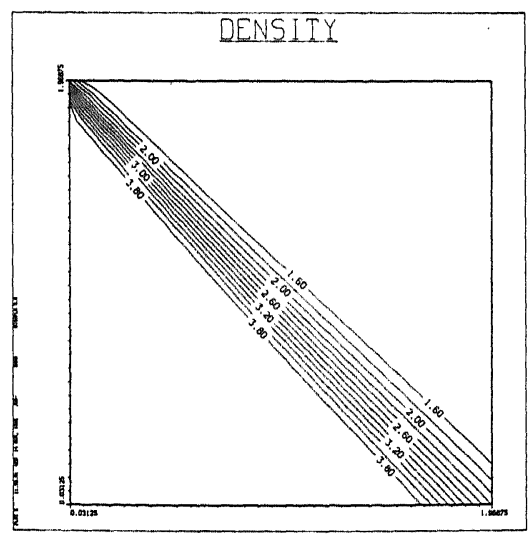

Figure 6c.

Density contours of a contact discontinuity on a $16 \times 16$ (figure 5) and a $32 \times 32$ (figure 6) mesh, obtained by the first order scheme and after 1 and $4 \mathrm{DeC}$ iteration steps. 